\title{
Old Drug Acquires New Target: Metformin and Sirt1
}

\section{Yuriy K Bashmakov* and Ivan M Petyaev}

Lycotec Ltd, Granta Park, Cambridge, UK

Type 2 diabetes mellitus [T2DM] is a progressive metabolic disease which requires multiple and frequent treatment plan adjustments during the course of the disease. Conventional therapeutic strategy usually starts with lifestyle interventions supported by prescription of one glucose-lowering drug. When insulin resistance progresses and the monotherapy option becomes insufficient to control plasma glucose, patients are usually switched to a double drug regimen. In the worst case scenario further progression of insulin resistance and decline in endogenous insulin production require administration of exogenous insulin which becomes an unavoidable therapeutic imperative for many T2DM patients [1]. Metformin is the oldest and most widely prescribed first choice anti-diabetic drug representing the group of guanidinebased hypoglycemic agents. Most newly diagnosed T2DM patients are placed on metformin monotherapy which is later supplemented or often substituted by sulphonylureas, thiazolidinediones and dipeptidyl peptidase-4 inhibitors [2].

The antidiabetic properties of guanidine-related compounds were acknowledged for many centuries by the widespread use of G. officinalis (also known as French lilac, Goat's rue or Italian Fitch) in diabetes care. However, in scientific terms, the hypoglycemic effect of guanidines, in particular the effect of isoamylene guanidine, was first demonstrated by C.K.Watanabe in 1918 [3]. The toxicity of guanidines imposed a significant limit on their pharmacological exploration, until a less toxic biguanidine analog - N,N-dimethylimidodicarbonimidic diamide (metformin) was synthesized in 1922. The discovery of insulin, whose hypoglycemic activity remains unsurpassed as well as the identification of other glucose-lowering compounds, weakened interest towards clinical use of guanidine-related compounds, including metformin, for decades. Therefore, it was not until 1979 that metformin was introduced in Europe and approved for T2DM use by the FDA in 1994.

The moderate hypoglycemic activity of metformin resulting in low risk of hypoglycemia is complemented by the wide spectrum of pharmacological activity of the drug. Besides controlling blood glucose, metformin is known to have some "pleiotropic effects" such as reduction of plasma free fatty acids, LDL and VLDL, promotion of body weight loss, decrease in cardiovascular mortality and morbidity, prevention of hepatosteatosis and some anti-neoplastic effect [4].

Despite significant clinical research on metformin use in T2DM patients, the molecular mechanisms underlying the anti-diabetic properties of the drug remain surprisingly obscure. It is believed though that hypoglycemic action of metformin reflects its direct effect on the hepatic gluconeogenic pathway and on glucose utilization in peripheral tissues. While most of the hepatic effects of metformin develop due to induction of AMP-activated protein kinase (AMPK) and/or changes in CREBP-binding protein phosphorylation, the effect of metformin on glucose uptake is rather related to enhanced expression of glucose transporters [5,6]. Recent reports have unveiled a variety of new molecular mechanisms to explain the anti-diabetic activity of metformin. Among exciting scientific findings there are newly emerging reports suggesting that metformin can mediate its anti-diabetic activity through the family of proteins called sirtuins. This piece of information comes from the report of Caton PW et al. [7] who described increased levels of SIRT1 protein and its activity in the livers of metformin-treated $d b / d b$ mice and HepG2 cells, a hepatocyte cell line. In addition, similar activation of SIRT1 has been reported this month in metformin-treated adipocytes by the same group of authors [8]. Although the effect of metformin on SIRT1 reportedly seems to be at least partially AMPK-mediated, these new findings suggest that metformin may exercise its pharmacological actions on diabetic phenotype via sirtuins, a family of $\mathrm{NAD}^{+}$-dependent Sir2 histone deacetylases. In mammalian cells the SIRT family is represented be at least seven proteins. Among them SIRT1 is the closest homolog of the yeast Sir2 protein implicated in aging, apoptosis and regulation of carbohydrate metabolism [9].

While independent confirmation and further expansion of the observations is undoubtedly required, the idea of cross-talk between metformin and SIRT-1 has a certain scientific sense. Both groups of agents - guanidines and SIRT-1 activators have a similarly moderate hypoglycemic effect and comparable ability to restore insulin sensitivity $[10,11]$. Moreover, there is a striking resemblance between the pleiotropic effects of metformin and the physiological features of SIRT1 activation [12]. In both cases reduced inflammation and oxidative stress, weight loss, improvement in plasma lipid profile as well as reduced cardiovascular mortality rate take place [13-16].

The growing body of evidence about the pleiotropic effects of metformin, which are likely to be sirtuin-mediated, sheds new light on the pharmacology of metformin and may lead to some reconsideration of drug regimens in T2DM patients. Metformin accounts for approximately $40 \%$ of all anti-diabetic drug dispensations and is used by nearly $45 \%$ of diabetic patients $[17,18]$. However, the real values for metformin use are much lower. Initial metformin nonadherence occurs in $16.6 \%$ patients [19], whereas poor compliance to the prescribed drug regimen takes place in one-third of all cases [20]. Although metformin is widely prescribed in newly diagnosed patients there is a conspicuous underrepresentation of the drug in the treatment regimen of patients with advanced T2DM [21]. An inadequate glycemic control and emerging safety considerations are the likely reasons for early metformin withdrawal. However, metformin safety concerns have been disputed recently by many authors claiming that advantages of metformin use surpass any of the potential risks. Indeed, the incidence of lactic acidosis and occurrence of acute renal failure are shown to be same in T2DM patients with or without metformin therapy [22]. Gastrointestinal discomfort symptoms and aggravation of neuropathy can be managed either by dose titration or supplementation with vitamin $B_{12}$ [23].

*Corresponding author: Yuriy K Bashmakovm, Lycotec Ltd, Granta Park Cambdridge, Cambridgeshire, CB1 6AL, UK, Tel: 1-972-9984307; E-mail: yuriy. bashmakov@lycotech.com

Received November 15, 2011; Accepted November 19, 2011; Published November 24, 2011

Citation: Bashmakov YK, Petyaev IM (2011) Old Drug Acquires New Target: Metformin and Sirt1. J Diabetes Metab 2:107e. doi:10.4172/2155-6156.1000107e

Copyright: ( 2011 Bashmakov YK, et al. This is an open-access article distributed under the terms of the Creative Commons Attribution License, which permits unrestricted use, distribution, and reproduction in any medium, provided the original author and source are credited. 
Citation: Bashmakov YK, Petyaev IM (2011) Old Drug Acquires New Target: Metformin and Sirt1. J Diabetes Metab 2:107e. doi:10.4172/2155$6156.1000107 e$

On the other hand, the pleiotropic effects of metformin appear to be conditional. It has been recently reported that the strongest antiinflammatory effect of metformin can be achieved in combination with fenofibrate [24]. Therefore it seems highly important to address in future the issue if other activators of SIRT1, including resveratrol and SIRT1 small molecule activators, can potentiate effects of metformin in T2DM patients.

In conclusion, systematic studies are required to answer the question of whether prolonged use of metformin beyond the point where the drug fails to control blood glucose has any measurable and significant health benefits through the activation of sirtuins.

\section{References}

1. Petznick A (2011) Insulin management of type 2 diabetes mellitus. Am Fam Physician 84:183-190.

2. Klarenbach S, Cameron C, Singh S, Ur E (2011) Cost-effectiveness of second-line antihyperglycemic therapy in patients with type 2 diabetes mellitus inadequately controlled on metformin. CMAJ183: 1213-1220.

3. Watanabe CK (1918) Studies in the metabolic changes induced by administration of guanidine bases. I. Influence of injected guanidine hydrochloride upon blood sugar content. J Biol Chem 33: 253-265

4. Anfossi G, Russo I, Bonomo K, Trovati M (2010) The cardiovascular effects of metformin: further reasons to consider an old drug as a cornerstone in the therapy of type 2 diabetes mellitus. Curr Vasc Pharmacol 8: 327-337.

5. Musi N (2006) AMP-activated protein kinase and type 2 diabetes. Curr Med Chem 13: 583-589.

6. Lee JO, Lee SK, Jung JH, Kim JH, You GY, et al. (2011) Metformin induces Rab4 through AMPK and modulates GLUT4 translocation in skeletal muscle cells. J Cell Physiol 226: 974-981.

7. Caton PW, Nayuni NK, Kieswich J, Khan NQ, Yaqoob MM, et al. (2010) Metformin suppresses hepatic gluconeogenesis through induction of SIRT1 and GCN5. J Endocrinol 205: 97-106.

8. Caton PW, Kieswich J, Yaqoob MM, Holness MJ, Sugden MC (2011) Metformin opposes impaired AMPK and SIRT1 function and deleterious changes in core clock protein expression in white adipose tissue of geneticallyobese db/db mice. Diabetes Obes Metab 13: 1097-1104.

9. Chung S, Yao H, Caito S, Hwang JW, Arunachalam G, et al. (2010) Regulation of SIRT1 in cellular functions: role of polyphenols. Arch Biochem Biophys 501 : 79-90.
10. Schwanstecher C, Schwanstecher M (2011) Targeting type 2 diabetes. Handb Exp Pharmacol: 1-33.

11. Timmers S, Konings E, Bilet L, Houtkooper RH, van de Weijer T, et al. (2011) Calorie Restriction-like Effects of 30 Days of Resveratrol Supplementation on Energy Metabolism and Metabolic Profile in Obese Humans. Cell Metab 14: 612-622.

12. Mouchiroud L, Molin L, Dallière N, Solari F (2010) Life span extension by resveratrol, rapamycin, and metformin: The promise of dietary restriction mimetics for an healthy aging. Biofactors 36: 377-382.

13. Panunti B, Kunhiraman B, Fonseca V (2005) The impact of antidiabetic therapies on cardiovascular disease. Curr Atheroscler Rep 7: 50-57.

14. Ghatak SB, Dhamecha PS, Bhadada SV, Panchal SJ (2011) Investigation of the potential effects of metformin on atherothrombotic risk factors in hyperlipidemic rats. Eur J Pharmacol 659: 213-223.

15. Ungvari Z, Labinskyy N, Mukhopadhyay P, Pinto JT, Bagi Z, et al. (2009) Resveratrol attenuates mitochondrial oxidative stress in coronary arterial endothelial cells. Am J Physiol Heart Circ Physiol 297: 1876-1881.

16. Purushotham A, Xu Q, Li X (2011) Systemic SIRT1 insufficiency results in disruption of energy homeostasis and steroid hormone metabolism upon highfat-diet feeding. FASEB J.

17. Patel H, Srishanmuganathan J, Car J, Majeed A (2007) Trends in the prescription and cost of diabetic medications and monitoring equipment in England 1991-2004. J Public Health (Oxf) 29: 48-52.

18. Aquilante CL, Zhang W, McCollum M (2007) Race, ethnicity, and use of thiazolidinediones among US adults with diabetes. Curr Med Res Opin 23 489-494.

19. Nichols GA, Conner C, Brown JB (2010) Initial nonadherence, primary failure and therapeutic success of metformin monotherapy in clinical practice. Curr Med Res Opin 26: 2127-2135.

20. Jaffiol C (2009) Current management of type 2 diabetes in France. Bull Acad Natl Med 193: 1645-1661.

21. DeFronzo RA, Abdul-Ghani M (2011) Type 2 diabetes can be prevented with early pharmacological intervention. Diabetes Care 34: 202-209.

22. Holstein A, Stumvoll M (2005) Contraindications can damage your health--is metformin a case in point? Diabetologia 48: 2454-2459.

23. Mahajan R, Gupta K (2010) Revisiting Metformin: Annual Vitamin B12 Supplementation may become Mandatory with Long-Term Metformin Use. J Young Pharm 2: 428-429.

24. Pruski M, Krysiak R, Okopien B (2009) Pleiotropic action of short-term metformin and fenofibrate treatment, combined with lifestyle intervention, in type 2 diabetic patients with mixed dyslipidemia. Diabetes Care 32: 1421-1424. 